\title{
A MODEL OF THE TEICHMÜLLER SPACE OF GENUS-ZERO BORDERED SURFACES BY PERIOD MAPS
}

\author{
DAVID RADNELL, ERIC SCHIPPERS, AND WOLFGANG STAUBACH
}

\begin{abstract}
We consider Riemann surfaces $\Sigma$ with $n$ borders homeomorphic to $\mathbb{S}^{1}$ and no handles. Using generalized Grunsky operators, we define a period mapping from the infinite-dimensional Teichmüller space of surfaces of this type into the unit ball in the linear space of operators on an $n$-fold direct sum of Bergman spaces of the disk. We show that this period mapping is holomorphic and injective.
\end{abstract}

\section{INTRODUCTION}

1.1. Introduction. The classical period mapping takes compact Riemann surfaces of genus $g$ into the Siegel upper half-plane, which consists of symmetric $g \times g$ matrices with positive-definite imaginary part. It is a classical fact that this map is holomorphic [10.

S. Nag [11] and S. Nag and D. Sullivan [12] constructed a period mapping of the universal Teichmüller space $T\left(\mathbb{D}^{+}\right)$, where $\mathbb{D}^{+}=\{z:|z|<1\}$. This period map takes the infinite-dimensional Teichmüller space into the Siegel disk of bounded operators $T$ on the Dirichlet space of the disk satisfying $\|T\|<1$. This is an alternate formulation of the Siegel upper half-plane of operators with positivedefinite imaginary part. L. Takhtajan and L.-P. Teo [23] later showed, remarkably, that the period mapping is in fact the Grunsky operator of univalent function theory [5,13, and gave the first complete proof that the period mapping is holomorphic.

In this paper, we generalize the period mapping to the case of the Teichmüller space of genus-zero surfaces with $n$ closed non-overlapping disks removed. The period mapping takes the Teichmüller space of this type into the direct product of the Teichmüller space of genus-zero surfaces with $n$ punctures and a space of bounded operators on an $n$-fold sum of Bergman spaces of the disk. The portion mapping into the Teichmüller space of punctured surfaces can of course be represented by period matrices using the classical method.

Our construction uses a generalized Grunsky operator, which was shown by the authors to be bounded by one [18, and thus lies in a kind of Siegel disk. We show that this mapping is holomorphic. The separation of the period mapping into a finite-dimensional part, involving compact surfaces with punctures,

Received by the editors October 18, 2017.

2010 Mathematics Subject Classification. Primary 30F60, 32G15, 32G20; Secondary 30C55, 30H20, 46G20.

The first author acknowledges the support of the Academy of Finland's project "Algebraic structures and random geometry of stochastic lattice models".

The second and third authors are grateful for the financial support from the Wenner-Gren Foundations. The second author was also partially supported by the National Sciences and Engineering Research Council of Canada. 
and an infinite-dimensional part, consisting of bounded operators on direct sums of Bergman spaces, relies on a fiber structure of Teichmüller space discovered by D. Radnell and E. Schippers [17. Holomorphicity of this fibration, and a resulting new set of complex coordinates [17, plays a key role in our proof of holomorphicity of the period map. The demonstration of this was accomplished using a variational technique of Radnell 14 which was obtained by modifying that of F. Gardiner and M. Schiffer [6, 10].

The period mapping of compact surfaces is known classically to be one of several non-trivially equivalent ways of placing a complex structure on Teichmüller space. Our construction extends this to another infinite-dimensional setting; namely, we show that the generalized period mapping defines a complex structure on the Teichmüller space of genus zero surfaces with boundary. Furthermore, it relates the complex structure to vector spaces of holomorphic objects on the surface, just as the classical period mapping relates the complex structure to the vector space of holomorphic one-forms. More explicitly, by [18, Theorem 4.1], the conformally invariant Dirichlet space of holomorphic functions on $\Sigma$ is the graph of the generalized period mapping. We expect that these results extend to the case of non-zero genus. Further algebraic and geometric properties of the period mapping remain to be explored.

1.2. Bergman spaces of one-forms. We establish some notation for Bergman spaces. Let $\Omega$ be a domain in $\overline{\mathbb{C}}$. Define $A^{2}(\Omega)_{\text {harm }}$ to be the set of harmonic one-forms $\alpha$ on $\Omega$ which are $L^{2}$ in the sense that

$$
\|\alpha\|^{2}=\frac{i}{2} \iint_{\Omega} \alpha \wedge \bar{\alpha}<\infty .
$$

We will call this the harmonic Bergman space. It has a natural inner product given by

$$
(\alpha, \beta)=\frac{i}{2} \iint_{\Omega} \alpha \wedge \bar{\beta}
$$

The subset of $A^{2}(\Omega)_{\text {harm }}$ consisting of holomorphic one-forms is the Bergman space which is denoted by $A^{2}(\Omega)$. We will represent one-forms in the Bergman space by functions. That is, if $\alpha$ is a one-form in $A^{2}(\Omega)$, then in $\Omega \backslash\{\infty\}$ it has a unique expression $h(z) d z$. In a neighborhood of $\infty$, using the chart $w \mapsto 1 / w, \alpha$ has the expression $\alpha=-w^{-2} h(1 / w) d w$.

The condition that $\alpha=h(z) d z \in L^{2}$ can then be expressed as follows. For some $r>1$, set $U=\{z:|z|<r\}$ and $V=\{z:|z|>1 / r\} \cup\{\infty\}$. Then $\alpha$ is in $A^{2}(\Omega)$ if and only if

$$
\iint_{\Omega \cap U}|h(z)|^{2} d A_{z}<\infty \text { and } \iint_{1 /(\Omega \cap V)}\left|w^{-2} h(1 / w)\right|^{2} d A_{w}<\infty .
$$

Here we use $d A_{z}$ as an abbreviation for $(d \bar{z} \wedge d z) / 2 i$, and $1 / D$ means $\{z \in \overline{\mathbb{C}}$ : $1 / z \in D\}$. If both conditions are satisfied, then

$$
\frac{i}{2} \iint_{\Omega} \alpha \wedge \bar{\alpha}=\iint_{\Omega \backslash\{\infty\}}|h(z)|^{2} d A_{z} .
$$

We will abbreviate the expression for the right-hand integral by

$$
\iint_{\Omega}|h(z)|^{2} d A_{z}
$$

although the reader should keep in mind the implicit condition on $h$ at $\infty$. 
If $\Omega$ is simply connected, then every $\alpha \in A^{2}(\Omega)_{\text {harm }}$ has a unique decomposition $\alpha=h(z) d z+\overline{g(z)} d \bar{z}$ for some holomorphic functions $g$ and $h$ in $A^{2}(\Omega)$. That is,

$$
A^{2}(\Omega)_{\text {harm }}=A^{2}(\Omega) \oplus \overline{A^{2}(\Omega)} .
$$

It is easily checked that this decomposition is orthogonal with respect to the inner product (1.1). Thus we have that

$$
\|h(z) d z+\overline{g(z)} d \bar{z}\|^{2}=\iint_{\Omega}\left(|h(z)|^{2}+|g(z)|^{2}\right) d A_{z} .
$$

We will also consider the space of exact one-forms in the harmonic or holomorphic Bergman space, which we denote by

$$
A_{e}^{2}(\Omega)=\left\{\alpha \in A^{2}(\Omega): \alpha=d H \text { for some holomorphic } H\right\}
$$

and similarly for $A_{e}^{2}(\Omega)_{\text {harm. }}$. In $A_{e}^{2}(\Omega)$, if we express $\alpha=h(z) d z$, there is some holomorphic function $H$ with domain $\Omega$ such that $H^{\prime}(z)=h(z)$. Holomorphicity on $\Omega$ means that $H$ is holomorphic on $\Omega \cap U$ and $H(1 / z)$ is holomorphic on $1 /(\Omega \cap V)$. If $\infty \in \Omega$, this implies in particular that $H$ has a finite limit as $z \rightarrow \infty$; equivalently, $H$ is continuous in the sphere topology. Note that the decomposition of $A^{2}(\Omega)_{\text {harm }}$ restricts to a decomposition $A_{e}^{2}(\Omega)_{\text {harm }}=A_{e}^{2}(\Omega) \oplus \overline{A_{e}^{2}(\Omega)}$, when $\Omega$ is simply connected.

Up to constants, the exact Bergman spaces are thus each isometric with a Dirichlet space. The harmonic Dirichlet space $\mathcal{D}(\Omega)_{\text {harm }}$ is the space of harmonic functions $H$ such that

$$
\frac{i}{2} \iint_{\Omega} d H \wedge \overline{d H}<\infty
$$

The Dirichlet space of holomorphic functions is denoted by $\mathcal{D}(\Omega)$. For $p \in \Omega$, $\mathcal{D}_{p}(\Omega)_{\text {harm }}$ denotes the subset of $\mathcal{D}(\Omega)_{\text {harm }}$ whose elements vanish at $p$, and similarly for $\mathcal{D}_{p}(\Omega)$. For simply connected domains $\Omega$, the elements of the harmonic Dirichlet space have a decomposition $H=F+\bar{G}$, where $F$ and $G$ are holomorphic, so that we can write

$$
\mathcal{D}_{p}(\Omega)_{\text {harm }}=\mathcal{D}_{p}(\Omega) \oplus \overline{\mathcal{D}_{p}(\Omega)} .
$$

Note that in $\mathcal{D}(\Omega)_{\text {harm }}$ the decomposition is not unique because constants are both holomorphic and antiholomorphic. We have the isometry

$$
\begin{aligned}
d: \mathcal{D}_{p}(\Omega)_{\text {harm }} & \longrightarrow A_{e}^{2}(\Omega)_{\text {harm }} \\
H & \longmapsto d H .
\end{aligned}
$$

The decompositions of $\mathcal{D}_{p}(\Omega)_{\text {harm }}$ and $A_{e}^{2}(\Omega)_{\text {harm }}$ commute with this isometry.

Let $f: D_{1} \rightarrow D_{2}$ be a biholomorphism between two domains $D_{1}, D_{2}$ in $\overline{\mathbb{C}}$. We have a pull-back operator defined by

$$
\begin{aligned}
& \hat{\mathcal{C}}_{f}: A^{2}\left(D_{2}\right)_{\text {harm }} \longrightarrow A^{2}\left(D_{1}\right)_{\text {harm }} \\
& h(z) d z+\overline{g(z)} d \bar{z} \longmapsto h \circ f(z) \cdot f^{\prime}(z) d z+\overline{g \circ f(z) \cdot f^{\prime}(z)} d \bar{z} .
\end{aligned}
$$

Clearly $\hat{\mathcal{C}}_{f}$ is an isometry, and it also restricts to an isometry from $A_{e}^{2}\left(D_{2}\right)_{\text {harm }}$ to $A_{e}^{2}\left(D_{1}\right)_{\text {harm }}$. Furthermore, it restricts to an isometry from $A^{2}\left(D_{2}\right)$ to $A^{2}\left(D_{1}\right)$ and from $A_{e}^{2}\left(D_{2}\right)$ to $A_{e}^{2}\left(D_{1}\right)$. 
The composition operator

$$
\begin{aligned}
\mathcal{C}_{f}: \mathcal{D}_{p}\left(D_{2}\right)_{\text {harm }} & \longrightarrow \mathcal{D}_{f(p)}\left(D_{1}\right)_{\text {harm }} \\
H & \longmapsto H \circ f
\end{aligned}
$$

is also an isometry, and we have that

$$
d \circ \mathcal{C}_{f}=\hat{\mathcal{C}}_{f} \circ d
$$

which incidentally motivates the notation $\hat{\mathcal{C}}_{f}$.

Remark 1.1 (Notation). Throughout the paper, operators without hats act on functions and operators with hats act on one-forms. We shall also denote the closure of a set $A$ by $A^{\mathrm{cl}}$, and its interior by $A^{\text {int }}$.

In the remainder of this paper, we will usually identify the elements $\alpha=h(z) d z$ of the holomorphic Bergman space with the function $h(z)$, except when emphasizing the fact that the elements are one-forms. The function is always written as a function of the standard coordinate $z$ in $\mathbb{C} \subset \overline{\mathbb{C}}$ rather than as a function of a coordinate at $\infty$.

We will not be directly working with Dirichlet spaces in this paper. They will be used only to apply results of the authors [18] for Dirichlet spaces to Bergman spaces, through the use of the isometry (1.4). These results involve a "reflection" of harmonic Dirichlet functions in quasidisks, obtained by extending to the boundary of the quasidisks, and then extending them to the complementary quasidisk. One may summarize the situation as follows: in the present paper, the use of one-forms creates a clearer geometric picture, whereas in the paper [18, the use of functions created a clearer analytic picture.

\section{GRunsky maP FOR MUltiply-CONNECTED DOMAins}

2.1. The generalized Faber and Grunsky operators. In this section we define certain generalizations of a Faber operator and the Grunsky operator to multiple maps with non-overlapping images. First we define the Faber operator and Grunsky operator associated with a single conformal map. For the concept of a Faber operator see P. Suetin 22]; for the Grunsky operator see for example [2,5, 13 .

Let $\Gamma$ be a Jordan curve not containing $\infty$, let $\Omega^{+}$be the bounded component of the complement of $\Gamma$ in $\overline{\mathbb{C}}$, and let $\Omega^{-}$be the other complementary component. Let

$$
\mathbb{D}^{+}=\{z:|z|<1\} \quad \text { and } \quad \mathbb{D}^{-}=\{z:|z|>1\} \cup\{\infty\} .
$$

Let $f: \mathbb{D}^{+} \rightarrow \Omega^{+}$be a conformal map. Following [18,19, we define the operators

$$
P\left(\Omega^{ \pm}\right): \mathcal{D}_{\text {harm }}\left(\Omega^{+}\right) \longrightarrow \mathcal{D}\left(\Omega^{ \pm}\right)
$$

by

$$
\left[P\left(\Omega^{ \pm}\right) h\right](z)= \pm \lim _{r \nearrow 1} \frac{1}{2 \pi i} \int_{f\left(C_{r}\right)} \frac{h(\zeta)}{\zeta-z} d \zeta, \quad z \in \Omega^{ \pm},
$$

where $C_{r}$ is the circle $\{w:|w|=r\}$ traced counterclockwise. Furthermore, define the map (the Faber operator)

$$
\begin{aligned}
\mathrm{I}_{f}: \mathcal{D}_{\infty}\left(\mathbb{D}^{-}\right) & \longrightarrow \mathcal{D}_{\infty}\left(\Omega^{-}\right) \\
h & \longmapsto P\left(\Omega^{-}\right) \mathcal{C}_{f-1} \mathbf{R} h,
\end{aligned}
$$

where $\mathbf{R}: \mathcal{D}_{\infty}\left(\mathbb{D}^{-}\right) \rightarrow \mathcal{D}_{0}\left(\mathbb{D}^{+}\right)$is given by $\mathbf{R} h(z)=h(1 / \bar{z})$. 
The limiting integral is necessary since Jordan curves are of course not in general rectifiable. The operators $P\left(\Omega^{ \pm}\right)$were shown to be well-defined maps which are bounded with respect to the Dirichlet seminorm (1.3). It was also shown in [19] that the Faber operator is an isomorphism precisely for quasicircles. This remarkable result is originally due to Y. Shen [21, with a somewhat different formulation of the operator; closely related results for convergence of Faber series on quasidisks were obtained by A. Çavuş [3].

We now consider the multiply-connected case. The following notation will be in force for the remainder of the paper. Let $\Sigma \subset \overline{\mathbb{C}}$ be a multiply-connected domain, which is bounded by $n$ non-overlapping quasicircles $\Gamma_{i}, i=1, \ldots, n$. We assume that $\infty \in \Sigma$. This normalization is a matter of convenience, and will be removed shortly. Let $\Omega_{i}^{+}$denote the component of the complement of $\Gamma_{i}$ in $\overline{\mathbb{C}}$ which does not intersect $\Sigma$, and let $\Omega_{i}^{-}$denote the other component of the complement of $\Gamma_{i}$. For each $i, \Omega_{i}^{-}$contains $\Sigma$; in fact

$$
\Sigma=\bigcap_{i=1}^{n} \Omega_{i}^{-} .
$$

We will also fix points $p_{i} \in \Omega_{i}^{+}$for $i=1, \ldots, n$.

For $i=1, \ldots, n$, fix conformal maps $f_{i}: \mathbb{D}^{+} \rightarrow \Omega_{i}^{+}$such that $f_{i}(0)=p_{i}$. Let $\boldsymbol{f}=\left(f_{1}, \ldots, f_{n}\right)$. In [18] the following generalized Faber operator was defined:

$$
\begin{aligned}
\mathbf{I}_{\boldsymbol{f}}: \bigoplus^{n} \mathcal{D}_{\infty}\left(\mathbb{D}^{-}\right) & \longrightarrow \mathcal{D}_{\infty}(\Sigma) \\
\left(h_{1}, \ldots, h_{n}\right) & \longmapsto \sum_{i=1}^{n} \mathrm{I}_{f_{i}} h_{i} .
\end{aligned}
$$

It was shown in [18] that this is an isomorphism. The generalized Grunsky operator was also defined:

$$
\boldsymbol{G r}(\boldsymbol{f})=\left(\mathrm{P}_{0}\left(\mathbb{D}^{+}\right) \mathcal{C}_{f_{1}} \mathbf{I}_{\boldsymbol{f}}, \ldots, \mathrm{P}_{0}\left(\mathbb{D}^{+}\right) \mathcal{C}_{f_{n}} \mathbf{I}_{\boldsymbol{f}}\right): \bigoplus^{n} \mathcal{D}_{\infty}\left(\mathbb{D}^{-}\right) \longrightarrow \bigoplus^{n} \mathcal{D}_{0}\left(\mathbb{D}^{+}\right)
$$

where

$$
\left[P_{0}\left(\mathbb{D}^{+}\right) H\right](z)=\left[P\left(\mathbb{D}^{+}\right) H\right](z)-\left[P\left(\mathbb{D}^{+}\right) H\right](0) .
$$

The blocks of this matrix (taking the $i$ th component to the $j$ th component of the direct sum) are denoted $\mathrm{Gr}_{j i}$. Note that this block depends only on $f_{i}$ and $f_{j}$ but for notational convenience we mostly write $\mathrm{Gr}_{j i}$ instead of $\mathrm{Gr}_{j i}\left(f_{i}, f_{j}\right)$. Further technical work was required to make sense of the composition $\mathcal{C}_{f_{j}} \mathbf{I}_{\boldsymbol{f}}$; this was accomplished in [18] and publications cited therein. Essentially, one may think of the composition operator as acting on boundary values of harmonic functions. In this paper, we will derive an equivalent integral formula and work directly with that.

Generalized Grunsky operators for non-overlapping mappings were considered by J. A. Hummel [8]. They are also considered in Takhtajan and Teo [23] in the case of a pair of non-overlapping maps whose images fill the sphere minus a quasicircle (that is, for a conformal welding pair).

We would like to use the equivalent form of the generalized Grunsky operator on exact one-forms rather than functions. Let

$$
\oplus^{n} d=(d, \ldots, d): \bigoplus^{n} \mathcal{D}_{0}\left(\mathbb{D}^{+}\right) \longrightarrow \bigoplus^{n} A^{2}\left(\mathbb{D}^{+}\right)
$$


and similarly define

$$
\oplus^{n} d^{-1}: \bigoplus^{n} A^{2}\left(\mathbb{D}^{-}\right) \longrightarrow \bigoplus^{n} \mathcal{D}_{\infty}\left(\mathbb{D}^{-}\right)
$$

Thus we may define

$$
\hat{\mathbf{I}}_{\boldsymbol{f}}=d \circ \mathbf{I}_{\boldsymbol{f}} \circ \oplus^{n} d^{-1}: \bigoplus^{n} A^{2}\left(\mathbb{D}^{-}\right) \longrightarrow A_{e}^{2}(\Sigma)
$$

and

$$
\widehat{\mathbf{G r}}(\boldsymbol{f})=\oplus^{n} d \circ \mathbf{G r}(\boldsymbol{f}) \circ \oplus^{n} d^{-1}: \bigoplus^{n} A^{2}\left(\mathbb{D}^{-}\right) \longrightarrow \bigoplus^{n} A^{2}\left(\mathbb{D}^{+}\right)
$$

with the blocks $\widehat{\operatorname{Gr}}_{j i}\left(f_{j}, f_{i}\right)$ similarly being defined as the block components of $\widehat{\operatorname{Gr}}(\boldsymbol{f})$. We will abbreviate these blocks as $\widehat{\mathrm{Gr}}_{j i}$. In the rest of the paper, we will use the Pythagorean norm on the direct sum $\bigoplus^{n} A^{2}\left(\mathbb{D}^{-}\right)$,

$$
\left\|\left(h_{1}, \ldots, h_{n}\right)\right\|_{\oplus^{n} A^{2}\left(\mathbb{D}^{-}\right)}^{2}=\sum_{k=1}^{n}\left\|h_{k}\right\|_{A^{2}\left(\mathbb{D}^{-}\right)}^{2},
$$

and similarly for $\bigoplus A^{2}\left(\mathbb{D}^{+}\right)$.

Remark 2.1. It can be shown that the graph of the Grunsky operator in $\mathcal{D}_{\infty}\left(\mathbb{D}^{+}\right) \oplus$ $\mathcal{D}_{0}\left(\mathbb{D}^{-}\right)$is the pull-back of the Dirichlet space $\mathcal{D}_{\infty}(\Sigma)$ under $\left(\mathcal{C}_{f_{1}}, \ldots, \mathcal{C}_{f_{n}}\right)$ [18]. It follows immediately from the fact that $\mathbf{I}_{\boldsymbol{f}}$ is an isomorphism that $\hat{\mathbf{I}}_{\boldsymbol{f}}$ is also an isomorphism. Using this fact we can interpret the graph of $\widehat{\mathbf{G r}}(\boldsymbol{f})$ as the pullback of $A_{e}^{2}(\Sigma)$ under $\left(\hat{\mathcal{C}}_{f_{1}}, \ldots, \hat{\mathcal{C}}_{f_{n}}\right)$, so long as we interpret $\hat{\mathcal{C}}_{f_{i}} \hat{\mathbf{I}}_{\boldsymbol{f}}$ as $d \mathcal{C}_{f_{i}} \mathbf{I}_{\boldsymbol{f}} d^{-1}$. Although we will not make use of this fact in our proofs, it is an important point for interpretation of the results of this paper.

Theorem 2.2 ([18]). Let $\Sigma \subseteq \overline{\mathbb{C}}$ be a domain containing $\infty$, bounded by $n$ nonintersecting quasicircles $\Gamma_{i}, i=1, \ldots, n$. Let $\Omega_{i}^{+}$and $\Omega_{i}^{-}$be the bounded and unbounded components of the complement of the quasicircle $\Gamma_{i}$, and let $\boldsymbol{f}=\left(f_{1}, \ldots, f_{n}\right)$ for conformal maps $f_{i}: \mathbb{D}^{+} \rightarrow \Omega_{i}^{+}, i=1, \ldots, n$. The Grunsky operator $\widehat{\mathbf{G r}}(\boldsymbol{f})$ satisfies $\|\widehat{\mathbf{G r}}(\boldsymbol{f})\|_{\oplus^{n} A^{2}\left(\mathbb{D}^{-}\right) \rightarrow \oplus^{n} A^{2}\left(\mathbb{D}^{+}\right)}<1$.

Proof. By a result of [18, the operator norm of $\mathbf{G r}$ is strictly bounded by one. The claim thus follows from the fact that $d: \mathcal{D}_{\infty}\left(\mathbb{D}^{-}\right) \rightarrow A^{2}\left(\mathbb{D}^{-}\right)$and $d: \mathcal{D}_{0}\left(\mathbb{D}^{+}\right) \rightarrow$ $A^{2}\left(\mathbb{D}^{+}\right)$are isometries.

2.2. Holomorphicity of $\widehat{\mathbf{G r}}(\boldsymbol{f})$ as a function of $\boldsymbol{f}$. Here we show that the operator $\widehat{\mathbf{G r}}(\boldsymbol{f})$ is holomorphic as a function of $\boldsymbol{f}=\left(f_{1}, \ldots, f_{n}\right)$. To do this, certain integral expressions for the components of $\widehat{\mathbf{G r}}(\boldsymbol{f})$ are required. First we define the antiholomorphic reflection

$$
\begin{aligned}
& \hat{\mathbf{R}}: A^{2}\left(\mathbb{D}^{-}\right) \\
& h(z) d z \longmapsto-\overline{A^{2}\left(\mathbb{D}^{+}\right)} \\
& h\left(\bar{z}^{-2} h(1 / \bar{z}) d \bar{z} .\right.
\end{aligned}
$$

This is an anti-isometry by change of variables.

Theorem 2.3. Let $\Sigma, \Omega_{i}^{ \pm}, p_{i}$, and $f_{i}$ be as above for $i=1, \ldots, n$. We have that for any $i \in\{1, \ldots, n\}$ and $\alpha(z)=h(z) d z \in A^{2}\left(\mathbb{D}^{-}\right)$

$$
\widehat{\mathrm{Gr}}_{i i} h(z)=\frac{1}{\pi} \iint_{\mathbb{D}^{+}}\left[\frac{1}{(\zeta-z)^{2}}-\frac{f_{i}^{\prime}(\zeta) f_{i}^{\prime}(z)}{\left(f_{i}(\zeta)-f_{i}(z)\right)^{2}}\right] \hat{\mathbf{R}} h(\zeta) d A_{\zeta} .
$$


Furthermore, for any $i, j \in\{1, \ldots, n\}$ such that $i \neq j$ we have

$$
\widehat{\operatorname{Gr}}_{j i} h(z)=\frac{1}{\pi} \iint_{\mathbb{D}^{+}} \frac{f_{i}^{\prime}(\zeta) f_{j}^{\prime}(z)}{\left(f_{i}(\zeta)-f_{j}(z)\right)^{2}} \hat{\mathbf{R}} h(\zeta) d A_{\zeta} .
$$

Proof. The first claim is [20, Theorem 4.13], and the second follows by differentiating [18, Theorem 4.5]. Differentiating under the integral sign is justified by the fact that the integrand is absolutely convergent, locally uniformly in $z$. To see this, observe that since $\left|f_{i}(\zeta)-f_{j}(z)\right| \geq M$ for $\zeta, z \in \mathbb{D}^{+}$, the Cauchy-Schwarz inequality yields that for any compact set $K \subset \mathbb{D}^{+}$and for all $z \in K$ one has

$$
\begin{aligned}
\frac{1}{\pi} \iint_{\mathbb{D}^{+}}\left|\frac{f_{i}^{\prime}(\zeta) f_{j}^{\prime}(z)}{\left(f_{i}(\zeta)-f_{j}(z)\right)^{2}} \hat{\mathbf{R}} h(\zeta)\right| d A_{\zeta} & \leq \frac{\left|f_{j}^{\prime}(z)\right|}{M^{2}}\left\|f_{i}^{\prime}\right\|_{A^{2}\left(\mathbb{D}^{+}\right)}\|\hat{\mathbf{R}} h\|_{A^{2}\left(\mathbb{D}^{+}\right)} \\
& \leq \frac{\left\|f_{j}^{\prime}\right\|_{L^{\infty}(K)}}{M^{2}}\left\|f_{i}^{\prime}\right\|_{A^{2}\left(\mathbb{D}^{+}\right)}\|h\|_{A^{2}\left(\mathbb{D}^{-}\right)}
\end{aligned}
$$

where we have also used the fact that $\hat{\mathbf{R}}$ is an isometry. Since $f_{i}\left(\mathbb{D}^{+}\right)$has finite area the claim follows.

Remark 2.4. Since $\hat{\mathbf{R}} h \in \overline{A^{2}\left(\mathbb{D}^{+}\right)}$, we could consider the Grunsky operator as a conjugate complex linear operator on $A^{2}\left(\mathbb{D}^{+}\right)$; see for example S. Bergman and M. Schiffer [2]. Inserting the reflection in the circle is natural in our interpretation of the Grunsky operator [20], and conveniently makes the operator complex linear on $A^{2}\left(\mathbb{D}^{-}\right)$.

The integral kernels in Theorem 2.3 are Möbius invariant, as we now show. For any $i, j \in\{1, \ldots, n\}$ (allowing $i=j$ ), it is easily seen that

$$
\frac{\left(T \circ f_{i}\right)^{\prime}(\zeta) \cdot\left(T \circ f_{j}\right)^{\prime}(z)}{\left(T \circ f_{i}(\zeta)-T \circ f_{j}(z)\right)^{2}}=\frac{f_{i}^{\prime}(\zeta) f_{j}^{\prime}(z)}{\left(f_{i}(\zeta)-f_{j}(z)\right)^{2}}
$$

for Möbius transformations $T$ of the form $T(z)=c z$ and $T(z)=z+b, b \in \mathbb{C}$, $c \in \mathbb{C} \backslash\{0\}$. For $T(z)=1 / z$, we compute

$$
\frac{\left(1 / f_{i}\right)^{\prime}(\zeta) \cdot\left(1 / f_{j}\right)^{\prime}(z)}{\left(1 / f_{i}(\zeta)-1 / f_{j}(z)\right)^{2}}=\frac{f_{i}^{\prime}(\zeta) f_{j}^{\prime}(z)}{\left(f_{i}(\zeta)-f_{j}(z)\right)^{2}} .
$$

Since the group of Möbius transformations is generated by these three types of transformations, the claim follows.

Thus, we can define the operator $\widehat{\mathbf{G r}}(\boldsymbol{f})$ for $\boldsymbol{f}=\left(f_{1}, \ldots, f_{n}\right)$ even when one of the quasidisks $f_{i}(\mathbb{D})$ contains $\infty$ in its closure by composing $f$ with a Möbius transformation (equivalently, by using the integral expression as a definition). Note that this also shows that the integral kernel of any block $\widehat{\mathrm{Gr}}_{j i}$ is non-singular on $\mathbb{D}^{+} \times \mathbb{D}^{+}$, regardless of whether $\infty$ is in the image of $f_{j}$ or $f_{i}$. With this extension of the definition to general $\boldsymbol{f}$, we have now shown the following.

Theorem 2.5. Let $\Sigma$ be an open subset of $\overline{\mathbb{C}}$, bounded by $n$ non-overlapping quasicircles $\Gamma_{i}$. Let $\Omega_{i}^{ \pm}, p_{i}$, and $f_{i}$ be as in Theorem 2.2 for $i=1, \ldots, n$. For any Möbius transformation $T$, denoting $\left(T \circ f_{1}, \ldots, T \circ f_{n}\right)$ by $T \circ \boldsymbol{f}$, we have

$$
\widehat{\mathbf{G r}}(T \circ \boldsymbol{f})=\widehat{\mathbf{G r}}(\boldsymbol{f}) .
$$

Furthermore the operator norm of $\widehat{\mathbf{G r}}(\boldsymbol{f})$ is strictly less than one. 
Remark 2.6. The operators $\hat{\mathbf{I}}_{\boldsymbol{f}}$ and $\hat{\mathcal{C}}_{\boldsymbol{f}_{i}}$ also extend to the case that $\infty \notin \Sigma$, so that the interpretation of $\widehat{\mathbf{G r}}(\boldsymbol{f})$ of Remark 2.1 continues to hold. Since this is not necessary for the proof of the main theorem (and indeed is fairly routine) we omit it.

We now require some definitions and results of Radnell and Schippers on nonoverlapping maps into Riemann surfaces with punctures [16]. Punctured Riemann surfaces will be denoted with a superscript $P$. Let

$$
A_{1}^{\infty}\left(\mathbb{D}^{+}\right)=\left\{\psi: \mathbb{D}^{+} \rightarrow \mathbb{C}: \psi \text { holomorphic, }\|\psi\|_{A_{1}^{\infty}\left(\mathbb{D}^{+}\right)}<\infty\right\},
$$

where

$$
\|\psi\|_{A_{1}^{\infty}\left(\mathbb{D}^{+}\right)}=\sup _{z \in \mathbb{D}^{+}}\left(1-|z|^{2}\right)|\psi(z)| .
$$

Let $\mathcal{O}^{\text {qc }}$ denote the set of injective conformal maps $g: \mathbb{D}^{+} \rightarrow \mathbb{C}$ such that $g(0)=0$ and $g$ is quasiconformally extendible to a map from $\overline{\mathbb{C}}$ to $\overline{\mathbb{C}}$. The map

$$
\begin{aligned}
\chi: \mathcal{O}^{\mathrm{qc}} & \longrightarrow \mathbb{C} \oplus A_{1}^{\infty}\left(\mathbb{D}^{+}\right) \\
g & \longmapsto\left(g^{\prime}(0), g^{\prime \prime} / g^{\prime}\right)
\end{aligned}
$$

is a bijection onto an open subset of the Banach space $\mathbb{C} \oplus A_{1}^{\infty}\left(\mathbb{D}^{+}\right)$with respect to the direct sum norm by [16, Theorem 3.1]. Thus $\mathcal{O}^{\text {qc }}$ inherits a complex structure by pull-back. We also let

$$
\mathcal{O}^{\mathrm{qc}}(n)=\left\{\boldsymbol{g}=\left(g_{1}, \ldots, g_{n}\right): g_{i} \in \mathcal{O}^{\mathrm{qc}} \text { for } i=1, \ldots, n\right\}
$$

which also has a complex structure obtained by taking the direct sum of $n$ copies of $\mathbb{C} \oplus A_{1}^{\infty}\left(\mathbb{D}^{+}\right)$, again with the direct sum norm. Finally,

Definition $2.7([16])$. Let $\Sigma^{P}$ be a compact Riemann surface with punctures $p_{1}, \ldots, p_{n}$. We define $\mathcal{O}^{\text {qc }}\left(\Sigma^{P}\right)$ to be the set of $n$-tuples $\boldsymbol{f}=\left(f_{1}, \ldots, f_{n}\right)$ of injective conformal maps $f_{i}: \mathbb{D}^{+} \rightarrow \Sigma^{P}$ such that for $i=1, \ldots, n$ the map $f_{i}$ has a quasiconformal extension to an open neighborhood of the closure of $\mathbb{D}^{+}, f_{i}(0)=p_{i}$, and $f_{i}\left(\mathbb{D}^{+}\right)^{\mathrm{cl}} \cap f_{j}\left(\mathbb{D}^{+}\right)^{\mathrm{cl}}$ is empty whenever $i \neq j$. We call $\boldsymbol{f} \in \mathcal{O}^{\mathrm{qc}}\left(\Sigma^{P}\right)$ a rigging of $\Sigma^{P}$.

In this article we are concerned with the special case that $\Sigma^{P}=\overline{\mathbb{C}} \backslash\left\{p_{1}, \ldots, p_{n}\right\}$.

Remark 2.8. Holomorphic maps and quasiconformal maps between punctured surfaces have unique holomorphic or quasiconformal continuations respectively to the compactifications. We will not distinguish notationally between these maps and their extensions. A punctured surface can be equivalently represented as a compact surface with marked points.

Remark 2.9. The following fact plays an important role ahead. In [16] we showed that $\mathcal{O}^{\mathrm{qc}}\left(\Sigma^{P}\right)$ has a natural complex structure in general. The local coordinates simplify in the special case that $\Sigma^{P}$ is the sphere with $n$ punctures $\overline{\mathbb{C}} \backslash\left\{p_{1}, \ldots, p_{n}\right\}$. By [16. Corollary 3.5], if we choose compact sets $K_{i}$ such that $p_{i}$ is in $K_{i}^{\text {int }}$ for each $i$, then

$$
V=\left\{\left(f_{1}, \ldots, f_{n}\right) \in \mathcal{O}^{\mathrm{qc}}\left(\Sigma^{P}\right): f_{i}(\mathbb{D})^{\mathrm{cl}} \subset K_{i}^{\mathrm{int}}, i=1, \ldots, n\right\}
$$

is open in $\mathcal{O}^{\mathrm{qc}}\left(\Sigma^{P}\right)$. Letting

$$
W=\left\{\left(f_{1}-p_{1}, \ldots, f_{n}-p_{n}\right):\left(f_{1}, \ldots, f_{n}\right) \in V\right\} \subseteq \mathcal{O}^{\mathrm{qc}}(n)
$$


and applying [16, Theorem 3.11] with coordinates $\zeta_{i}(z)=z-p_{i}$ the map

$$
\begin{aligned}
G: W & \longrightarrow V \\
\left(g_{1}, \ldots, g_{n}\right) & \longmapsto\left(g_{1}+p_{1}, \ldots, g_{n}+p_{n}\right)
\end{aligned}
$$

is a biholomorphism.

Let

$$
\mathfrak{B}(n)=\left\{T: \bigoplus A^{2}\left(\mathbb{D}^{-}\right) \longrightarrow \bigoplus^{n} A^{2}\left(\mathbb{D}^{+}\right):\|T\|<\infty\right\} .
$$

Recall that we are using the Pythagorean norm on $\bigoplus^{n} A^{2}\left(\mathbb{D}^{ \pm}\right)$.

Remark 2.10. In addressing holomorphic dependence of the Grunsky operator on the rigging $f$ below, we will need the following elementary observation. Let

$$
T: \bigoplus^{n} A^{2}\left(\mathbb{D}^{-}\right) \longrightarrow \bigoplus^{n} A^{2}\left(\mathbb{D}^{+}\right)
$$

be a linear operator and let $T_{j k}: A^{2}\left(\mathbb{D}^{-}\right) \rightarrow A^{2}\left(\mathbb{D}^{+}\right)$be its blocks. Using the inequality $\sup _{1 \leq k \leq n}\left|a_{k}\right| \leq \sqrt{\sum_{k=1}^{n}\left|a_{k}\right|^{2}} \leq \sum_{k=1}^{n}\left|a_{k}\right|$, we obtain for $h=\left(h_{1}, \ldots, h_{n}\right)$ the inequality

$$
\begin{aligned}
\|T\| & \leq \sup _{\substack{h \in \bigoplus^{n} A^{2}\left(\mathbb{D}^{-}\right) \\
\|h\| \leq 1}} \sum_{k=1}^{n}\left\|\sum_{j=1}^{n} T_{j k} h_{j}\right\|_{A^{2}\left(\mathbb{D}^{+}\right)} \\
& \leq \sup _{\substack{h \in \bigoplus^{n} A^{2}\left(\mathbb{D}^{-}\right) \\
\left\|h_{j}\right\| \leq 1, j=1, \ldots, n}} \sum_{k=1}^{n} \sum_{j=1}^{n}\left\|T_{j k} h_{j}\right\|_{A^{2}\left(\mathbb{D}^{+}\right)} .
\end{aligned}
$$

Thus, consider a family $T(t)$ of operators in $\mathfrak{B}(n)$ depending on a complex parameter $t$. To show that $T(t)$ is Gâteaux holomorphic at $t=0$, it is enough to show that

$$
\lim _{t \rightarrow 0}\left\|t^{-1}\left(T_{j k}(t)-T_{j k}(0)-t B_{j k}\right)\right\|=0
$$

for some $B: \bigoplus^{n} A^{2}\left(\mathbb{D}^{-}\right) \rightarrow \bigoplus^{n} A^{2}\left(\mathbb{D}^{+}\right)$with blocks $B_{j k}$.

Theorem 2.11. Fix distinct points $p_{1}, \ldots, p_{n} \in \mathbb{C}$ and let $K_{i}$ be non-intersecting compact sets such that $p_{i}$ are in $K_{i}^{\text {int }}$ for $i=1, \ldots, n$. Let $N=N_{1} \times \cdots \times N_{n}$, where $N_{i} \subseteq \mathbb{C}$ are open neighborhoods of 0 such that the sets $K_{i}+z_{i}$ are non-intersecting for all $\left(z_{1}, \ldots, z_{n}\right) \in N$. Let $W=\left\{\left(g_{1}, \ldots, g_{n}\right) \in \mathcal{O}^{\mathrm{qc}}(n): g_{i}(\mathbb{D})^{\mathrm{cl}}+p_{i} \subset K_{i}^{\text {int }}\right\}$. The map

$$
\begin{aligned}
H: W \times N & \longrightarrow \mathfrak{B}(n) \\
\left(g_{1}, \ldots, g_{n}, a_{1}, \ldots, a_{n}\right) & \longmapsto \widehat{\mathbf{G r}}\left(g_{1}+a_{1}+p_{1}, \ldots, g_{n}+a_{n}+p_{n}\right)
\end{aligned}
$$

is holomorphic.

Proof. By [4, p. 198] it is enough to show that $H$ is locally bounded and Gâteaux holomorphic. By Theorem $2.2 \widehat{\mathbf{G r}}(\boldsymbol{f})$ is bounded, so only Gâteaux holomorphicity remains. By Hartogs' theorem in the Banach space setting [9] it is enough to prove holomorphicity on $W$ and $N$ separately. Since $W$ is a subset of $\mathcal{O}^{\text {qc }}(n)=\bigoplus^{n} \mathcal{O}^{\text {qc }}$ we further reduce the problem to proving holomorphicity on the individual copies of $\mathcal{O}^{\text {qc }}$. Recall that the complex structure on $\mathcal{O}^{\text {qc }}$ is given by the pull-back of 
the complex structure on $\mathbb{C} \oplus A_{1}^{\infty}\left(\mathbb{D}^{+}\right)$under $g \mapsto\left(g^{\prime}(0), g^{\prime \prime} / g^{\prime}\right)$ (see equation (2.3) $)$. So holomorphicity on $W$ has been finally reduced to Gâteaux holomorphicity separately on $\mathbb{C}$ and $A_{1}^{\infty}\left(\mathbb{D}^{+}\right)$.

Note that Remark 2.10 yields that the holomorphicity of $\widehat{\mathbf{G r}}(\boldsymbol{f})$ follows from the holomorphicity of its blocks $\widehat{\operatorname{Gr}}_{k l}$ for $k, l=1, \ldots, n$. Recall that the block $\widehat{\operatorname{Gr}}_{k l}(\boldsymbol{f})$ is only a function of $f_{k}$ and $f_{l}$.

We first look at the diagonal components $\widehat{\mathrm{Gr}}_{i i}$. For fixed $a_{1}, \ldots, a_{n} \in N$, holomorphic dependence of $\widehat{G r}_{i i}$ on $A_{1}^{\infty}\left(\mathbb{D}^{+}\right)$is due to Takhtajan and Teo 23, Theorem B.1, p. 109] (note that there they use the integral formula of Theorem 2.3 as the definition of the operator). Let $f_{i}=g_{i}+a_{i}+p_{i}$. Since $\widehat{\mathrm{Gr}}_{i i}$ is invariant under $f_{i} \mapsto c f_{i}$ for $c \neq 0$ it is independent of $g^{\prime}(0)=f^{\prime}(0)$, and so $\widehat{\mathrm{Gr}}_{i i}$ is holomorphic on $\mathbb{C}$. For holomorphicity on $N$, one needs only to observe that $\widehat{\mathrm{Gr}}_{i i}$ is independent of $\left(a_{1}, \ldots, a_{n}\right) \in N$.

Now we prove that the off-diagonal components of $\widehat{\mathbf{G r}}(\boldsymbol{f})$ are holomorphic. First we fix $\left(a_{1}, \ldots, a_{n}\right) \in N$ and prove Gâteaux holomorphicity on $W$. Fix $j \in\{1, \ldots, n\}$. We will prove Gâteaux holomorphicity on the $j$ th copy of $\mathcal{O}^{\text {qc }}$. This requires only looking at the blocks $\widehat{\mathrm{Gr}}_{j i}$ and $\widehat{\mathrm{Gr}}_{i j}$ for $i \neq j$.

Fix $\left(g_{1}^{0}, \ldots, g_{n}^{0}\right) \in W$, and consider the complex lines $\left(q(t), \psi^{t}\right) \in \mathbb{C} \oplus A_{1}^{\infty}\left(\mathbb{D}^{+}\right)$, where $\psi^{t}=\left(g_{j}^{0}\right)^{\prime \prime} /\left(g_{j}^{0}\right)^{\prime}+t \phi$ for some $\phi \in A_{1}^{\infty}\left(\mathbb{D}^{+}\right)$and $q(t)=\left(g_{j}^{0}\right)^{\prime}(0)+c t$ for some $c \in \mathbb{C}$. Now define the curve $g_{j}^{t} \in \mathcal{O}^{\mathrm{qc}}$ to be the solution of the differential equation $\left(g_{j}^{t}\right)^{\prime \prime} /\left(g_{j}^{t}\right)^{\prime}=\psi^{t}$ with initial conditions $g_{j}^{t}(0)=0$ and $\left(g_{j}^{t}\right)^{\prime}(0)=q(t)$. That is, the curve $g_{j}^{t}$ corresponds to the above complex line under the map $\chi$ defined in (2.3). Since $\chi\left(\mathcal{O}^{\mathrm{qc}}\right) \subseteq \mathbb{C} \oplus A_{1}^{\infty}\left(\mathbb{D}^{+}\right)$is open, there is an $r>0$ such that $g_{j}^{t} \in \mathcal{O}^{\text {qc }}$ for all $|t|<r$.

Let $f_{i}^{0}=g_{i}^{0}+a_{i}+p_{i}$ for $i=1, \ldots, n$ and let $f_{j}^{t}=g_{j}^{t}+a_{i}+p_{i}$. Using Theorem 2.3. we now prove Gâteaux holomorphicity by proving that for all $i \neq j, t \mapsto \widehat{\operatorname{Gr}}_{j i}^{t}$ and $t \mapsto \widehat{\mathrm{Gr}}_{i j}^{t}$ are holomorphic in a neighborhood of $t=0$ in $\mathbb{C}$, where

$$
\widehat{\mathrm{Gr}}_{j i}^{t} h(z)=\widehat{\operatorname{Gr}}_{j i}\left(f_{j}^{t}, f_{i}^{0}\right) h(z)=\frac{1}{\pi} \iint_{\mathbb{D}^{+}} \frac{\left(f_{i}^{0}\right)^{\prime}(\zeta)\left(f_{j}^{t}\right)^{\prime}(z)}{\left(f_{i}^{0}(\zeta)-f_{j}^{t}(z)\right)^{2}} \hat{\mathbf{R}} h(\zeta) d A_{\zeta}
$$

and

$$
\widehat{\operatorname{Gr}}_{i j}^{t} h(z)=\widehat{\operatorname{Gr}}_{i j}\left(f_{i}^{0}, f_{j}^{t}\right) h(z)=\frac{1}{\pi} \iint_{\mathbb{D}^{+}} \frac{\left(f_{j}^{t}\right)^{\prime}(\zeta)\left(f_{i}^{0}\right)^{\prime}(z)}{\left(f_{j}^{t}(\zeta)-f_{i}^{0}(z)\right)^{2}} \hat{\mathbf{R}} h(\zeta) d A_{\zeta} .
$$

Let $L_{1}^{t}(z, \zeta):=\frac{\left(f_{i}^{0}\right)^{\prime}(\zeta)\left(f_{j}^{t}\right)^{\prime}(z)}{\left(f_{i}^{0}(\zeta)-f_{j}^{t}(z)\right)^{2}}$ and $L_{2}^{t}(z, \zeta):=\frac{\left(f_{j}^{t}\right)^{\prime}(\zeta)\left(f_{i}^{0}\right)^{\prime}(z)}{\left(f_{j}^{t}(\zeta)-f_{i}^{0}(z)\right)^{2}}$.

To prove the holomorphicity of $\widehat{\mathrm{Gr}}_{j i}^{t}$ we observe that, for fixed $z, \zeta \in \mathbb{D}^{+}, L_{1}^{t}(z, \zeta)$ is a holomorphic function of $t$ in a neighborhood of 0 . This follows from the fact that $f_{j}^{t}(z)$ is holomorphic in $t$ for fixed $z$ (by construction; see [16, p. 287] for an explicit expression). Now choose $\delta>0$ so that $\delta<r$. Then using Cauchy's integral formula we have for all $|t|<\delta$

$$
\begin{gathered}
L_{1}^{t}(z, \zeta)-L_{1}^{0}(z, \zeta)-\left.t \frac{d}{d t}\right|_{t=0} L_{1}^{t}(z, \zeta) \\
=\frac{t^{2}}{2 \pi i} \oint_{|s|=\delta} \frac{L_{1}^{s}(z, \zeta)}{s^{2}(s-t)} d s .
\end{gathered}
$$


Setting $\overline{u(z)}=\hat{\mathbf{R}} h$ and using the equality above together with the fact that $\hat{\mathbf{R}}$ is an isometry, we obtain

$$
\begin{aligned}
& \left\|\frac{\widehat{\mathrm{Gr}}_{j i}^{t}-\widehat{\mathrm{Gr}}_{j i}^{0}}{t}-\left.\frac{d}{d t}\right|_{t=0} \widehat{\mathrm{Gr}}_{j i}^{t}\right\| \|_{A^{2}\left(\mathbb{D}^{-}\right) \rightarrow A^{2}\left(\mathbb{D}^{+}\right)} \\
& \quad=\sup _{\|h\|_{A^{2}\left(\mathbb{D}^{-}\right)}=1}\left\|\left(\frac{\widehat{\mathrm{Gr}}_{j i}^{t}(f)-\widehat{\mathrm{Gr}}_{j i}^{0}(f)}{t}-\left.\frac{d}{d t}\right|_{t=0} \widehat{\mathrm{Gr}}_{j i}^{t}(f)\right) h\right\|_{A^{2}\left(\mathbb{D}^{+}\right)} \\
& =\frac{|t|}{2 \pi^{2}} \sup _{\|u\|_{A^{2}\left(\mathbb{D}^{+}\right)}=1}\left(\iint_{\mathbb{D}^{+}}\left|\iint_{\mathbb{D}^{+}}\left(\oint_{|s|=\delta} \frac{L_{1}^{s}(z, \zeta)}{s^{2}(s-t)} d s\right) \overline{u(\zeta)} d A_{\zeta}\right|^{1 / 2} d A_{z}\right)^{1 / 2} .
\end{aligned}
$$

To reduce expression size, temporarily let

$$
I(u ; s, z)=\iint_{\mathbb{D}^{+}} L_{1}^{s}(z, \zeta) \overline{u(\zeta)} d A_{\zeta} .
$$

By Fubini's theorem and the Cauchy-Schwarz inequality (in the contour integral), we see that

$$
\begin{aligned}
& \left\|\frac{{\widehat{\mathrm{Gr}_{j i}}}_{j i}^{t}-{\widehat{\mathrm{Gr}_{j i}}}^{0}}{t}-\left.\frac{d}{d t}\right|_{t=0} \widehat{\mathrm{Gr}}_{j i}^{t}\right\| \|_{A^{2}\left(\mathbb{D}^{-}\right) \rightarrow A^{2}\left(\mathbb{D}^{+}\right)} \\
& \quad=\frac{|t|}{2 \pi^{2}} \sup _{\|u\|_{A^{2}\left(\mathbb{D}^{+}\right)}=1}\left(\iint\left|\oint_{|s|=\delta} \frac{I(u ; s, z)}{s^{2}(s-t)} d s\right|^{2} d A_{z}\right)^{1 / 2} \\
& \quad \leq \frac{|t|}{2 \pi^{2}} \sup _{\|u\|_{A^{2}\left(\mathbb{D}^{+}\right)}=1}\left(\iint_{\mathbb{D}^{+}}\left(\oint_{|s|=\delta} \frac{|d s|}{|s|^{4}|s-t|^{2}}\right)\left(\oint_{|s|=\delta}|I(u ; s, z)|^{2}|d s|\right) d A_{z}\right)^{1 / 2} \\
& \quad \leq \frac{|t|}{2 \pi^{2}}\left(\oint_{|s|=\delta} \frac{|d s|}{|s|^{4}|s-t|^{2}}\right)^{1 / 2} \sup _{\|u\|_{A^{2}\left(\mathbb{D}^{+}\right)=1}}\left(\oint_{|s|=\delta}\|I(u ; s, z)\|_{L^{2}\left(\mathbb{D}^{+}\right)}^{2}|d s|\right)^{1 / 2} .
\end{aligned}
$$

Now we claim that for $|s|=\delta$ the operator with kernel $L_{1}^{s}(z, \zeta)$ is bounded on $L^{2}\left(\mathbb{D}^{+}\right)$, with a norm that depends only on $\delta$. To see this we observe that $f_{j}^{0}\left(\mathbb{D}^{+}\right)$ and $f_{i}^{0}\left(\mathbb{D}^{+}\right)$have disjoint closures; furthermore, on any holomorphic curve through $f_{j}^{0}$ we can ensure that the closures of the images remain in fixed disjoint sets for sufficiently small $t$ [16. Corollary 3.5]. As a consequence, for $|s|=\delta$ there is a constant $A_{\delta}>0$ such that $\left|f_{i}^{0}(\zeta)-f_{j}^{s}(z)\right|>A_{\delta}$. Furthermore the image of $f_{i}^{0}$ and $f_{j}^{s}$ (for fixed $s$ ) are both bounded, so $\left\|f_{i}^{0^{\prime}}\right\|_{A^{2}\left(\mathbb{D}^{+}\right)}$and $\left\|f_{j}^{s^{\prime}}\right\|_{A^{2}\left(\mathbb{D}^{+}\right)}$(for fixed $s$ ) are bounded. Again applying [16, Corollary 3.5] the image of $f_{j}^{s}$ is contained in a disk in $\mathbb{C}$ of radius independent of $s$ so the bound for $\left\|f_{j}^{s^{\prime}}\right\|_{A^{2}\left(\mathbb{D}^{+}\right)}$can be chosen uniformly in $s$. Therefore, there exist constants $B_{\delta}>0$ and $C_{\delta}>0$ such that

$$
\left\{\iint_{\mathbb{D}^{+}} \iint_{\mathbb{D}^{+}}\left|L_{1}^{s}(z, \zeta)\right|^{2} d A_{z} d A_{\zeta}\right\}^{1 / 2} \leq B_{\delta}\left\|\left(f_{i}^{0}\right)^{\prime}\right\|_{A^{2}\left(\mathbb{D}^{+}\right)}\left\|\left(f_{j}^{s}\right)^{\prime}\right\|_{A^{2}\left(\mathbb{D}^{+}\right)} \leq C_{\delta} .
$$


Now since the operator-norm of the integral operator with kernel $L_{1}^{s}(z, \zeta)$ (as a bounded linear operator from $L^{2}\left(\mathbb{D}^{+}\right)$to itself) is bounded by the left-hand side of (2.6), the claim follows.

Finally for $|t|<\delta$, applying the previous two estimates, we obtain

$$
\begin{aligned}
& \left\|\frac{\widehat{\mathrm{Gr}}_{j i}^{t}-\widehat{\mathrm{Gr}}_{j i}^{0}}{t}-\left.\frac{d}{d t}\right|_{t=0}{\widehat{\mathrm{Gr}_{j i}}}_{j i}^{t}\right\| \|_{A^{2}\left(\mathbb{D}^{-}\right) \rightarrow A^{2}\left(\mathbb{D}^{+}\right)} \\
& \quad \leq C_{\delta}|t|\left(\oint_{|s|=\delta} \frac{|d s|}{|s|^{4}|s-t|^{2}} \oint_{|s|=\delta\|u\|_{A^{2}\left(\mathbb{D}^{+}\right)=1}}\|u\|_{A^{2}\left(\mathbb{D}^{+}\right)}^{2}|d s|\right)^{1 / 2} \\
& \quad \leq C_{\delta}^{\prime}|t|\left(\oint_{|s|=\delta} \frac{2 \pi \delta|d s|}{|s|^{4}|s-t|^{2}}\right)^{1 / 2} \\
& \leq C_{\delta}^{\prime \prime} \frac{|t|}{\delta-|t|},
\end{aligned}
$$

which can be made as small as we like, provided $t$ is chosen small enough. This establishes the Gâteaux holomorphicity of $\widehat{\mathrm{Gr}}_{j i}^{t}$ in the first component of $W \times N$. In the second component, the proof above can be used in the same way: the integral kernel is holomorphic in $a_{j}$ under $f_{j}^{0} \mapsto f_{j}^{0}+a_{j}$, so one only needs to establish local boundedness. By the hypotheses on $N,\left|f_{i}^{0}(\zeta)-f_{j}^{0}(z)\right|$ is still uniformly bounded and the integral estimate (2.6) continues to hold. Proceeding as above we obtain holomorphicity in the second component.

The proof of holomorphicity of $\widehat{\mathrm{Gr}}_{i j}^{t}$ is the same as the above, except that one replaces the $L^{2}\left(\mathbb{D}^{+}\right)$boundedness of the integral operator with kernel $L_{1}^{t}(z, \zeta)$ with $L^{2}$ boundedness of the integral operator with kernel $L_{2}^{t}(z, \zeta)$. This ends the proof of the theorem.

\section{PERIOD MAP}

3.1. Fibration of the Teichmüller space of bordered surfaces. In this section we recall some definitions of Teichmüller space and rigged Teichmüller space. We require some results of Radnell and Schippers 17 on a fibration of Teichmüller space of surfaces with $n$ borders over the Teichmüller space of surfaces with $n$ punctures, which play a central role in the formulation of the period mapping and proof of its holomorphicity.

We say that a Riemann surface is a bordered surface of type $(g, n)$ if it is a Riemann surface of genus $g$ with $n$ boundary curves homeomorphic to $\mathbb{S}^{1}$. More precisely, we assume that the double of the Riemann surface $\Sigma^{D}$ is of genus $2 g+n-1$ and the ideal boundary $\partial \Sigma$ consists of $n$ closed analytic curves in $\Sigma^{D}$ each of which is homeomorphic to $\mathbb{S}^{1}$ with respect to the topology inherited from $\Sigma^{D}$. We note that such a Riemann surface $\Sigma$ is a bordered surface in the sense of Ahlfors and Sario 11. That is, there is an atlas of charts including boundary charts of the following form. Any point of the boundary is contained in a relatively open subset $U$ of the closure $\Sigma \cup \partial \Sigma$ such that there is a biholomorphism $\phi: U \rightarrow V$, where $V$ is a relatively open subset of the upper half-plane $\overline{\mathbb{H}}=\{z \in \mathbb{C}: \operatorname{Im}(z) \geq 0\}$ and $\phi(U \cap \partial \Sigma)$ is an open interval on $\operatorname{Im}(z)=0$. We assume that the transition functions $\phi_{1} \circ \phi_{2}^{-1}$ of any pair of charts are biholomorphic on their domain of definition. In the case that both are 
boundary charts, this means that maps $\phi_{1} \circ \phi_{2}^{-1}$ have biholomorphic extensions to an open set in $\mathbb{C}$ containing the original domain of definition of $\phi_{1} \circ \phi_{2}^{-1}$.

A quasiconformal map $f: \Sigma \rightarrow \Sigma_{1}$ between bordered Riemann surfaces of type $(g, n)$ must have an extension to the ideal boundary $\partial \Sigma$. We will not distinguish this extension notationally from the map on $\Sigma$. We say that quasiconformal maps $f: \Sigma \rightarrow \Sigma$ and $g: \Sigma \rightarrow \Sigma$ are homotopic rel boundary if they are homotopic via a homotopy $H:[0,1] \times \Sigma \rightarrow \Sigma$ such that $H(t, z)=f(z)=g(z)$ for all $z \in \partial \Sigma$ and $t \in[0,1]$.

We now define the Teichmüller space of such a Riemann surface.

Definition 3.1. Let $\Sigma$ be a Riemann surface whose universal cover is the unit disk. The Teichmüller space of $\Sigma$ is

$$
T(\Sigma)=\left\{\left(\Sigma, f, \Sigma_{1}\right)\right\} / \sim
$$

where $f: \Sigma \rightarrow \Sigma_{1}$ is quasiconformal and $\left(\Sigma, f_{1}, \Sigma_{1}\right) \sim\left(\Sigma, f_{2}, \Sigma_{2}\right)$ if and only if there is a biholomorphism $\sigma: \Sigma_{1} \rightarrow \Sigma_{2}$ such that $f_{2}^{-1} \circ \sigma \circ f_{1}$ is homotopic to the identity rel boundary. Denote the equivalence class of a triple $\left(\Sigma, f, \Sigma_{1}\right)$ by $\left[\Sigma, f, \Sigma_{1}\right]$.

In 14 Radnell defined a "rigged Teichmüller space" of a punctured surface, which was shown by Radnell and Schippers [15] to be intermediate between the Teichmüller space of a bordered surface and that of the compact surface obtained by sewing disks on the boundary. The rigged Teichmüller space and its relation to the usual Teichmüller space are instrumental in the proof of the main theorem.

Definition 3.2. Let $\Sigma_{0}^{P}$ be a compact surface with punctures $p_{1}, \ldots, p_{n}$. The rigged Teichmüller space of $\Sigma_{0}^{P}$ is

$$
\widetilde{T}\left(\Sigma_{0}^{P}\right)=\left\{\left(\Sigma_{0}^{P}, F_{1}, \Sigma_{1}^{P}, \boldsymbol{f}\right): F_{1}: \Sigma_{0}^{P} \rightarrow \Sigma_{1}^{P} \text { quasiconformal, } \boldsymbol{f} \in \mathcal{O}^{\mathrm{qc}}\left(\Sigma_{1}^{P}\right)\right\} / \sim,
$$

where $\sim$ is an equivalence relation defined by $\left(\Sigma_{0}^{P}, F_{1}, \Sigma_{1}^{P}, \boldsymbol{f}\right) \sim\left(\Sigma_{0}^{P}, F_{2}, \Sigma_{2}^{P}, \boldsymbol{g}\right)$ whenever there is a conformal map $\sigma: \Sigma_{1}^{P} \rightarrow \Sigma_{2}^{P}$ preserving the punctures and their order such that $F_{2}^{-1} \circ \sigma \circ F_{1}$ is homotopic to the identity (in such a way that the homotopy is constant on the punctures) and $g_{i}=\sigma \circ f_{i}$ for all $i=1, \ldots, n$.

There is in general a holomorphic fibration of the Teichmüller space of a bordered surface over the rigged Teichmüller space of a punctured surface. We need this in the special case that $\Sigma_{0}$ is $\overline{\mathbb{C}}$ minus disks. Fix disks $D_{i}=\left\{z:\left|z-p_{i}\right|<r_{i}\right\}$, $i=1, \ldots, n$, such that $D_{i}^{\mathrm{cl}} \cap D_{j}^{\mathrm{cl}}$ is empty whenever $i \neq j$. Set $\Sigma_{0}=\overline{\mathbb{C}} \backslash \bigcup_{i=1}^{n} D_{i}^{\mathrm{cl}}$ and $\Sigma_{0}^{P}=\overline{\mathbb{C}} \backslash\left\{p_{1}, \ldots, p_{n}\right\}$. Finally, fix $\boldsymbol{\tau}=\left(\tau_{1}, \ldots, \tau_{n}\right)$, where for each $i$ the map $\tau_{i}: \mathbb{D}^{+} \rightarrow D_{i}$ is a conformal bijection such that $\tau_{i}(0)=p_{i}$.

Now let $\left[\Sigma_{0}, F_{1}, \Sigma_{1}\right] \in T\left(\Sigma_{0}\right)$. Let $\mu\left(F_{1}\right)$ be the Beltrami differential of $F_{1}$ on $\Sigma_{0}$. Extend $\mu\left(F_{1}\right)$ to a Beltrami differential on $\Sigma_{0}^{P}$ by setting

$$
\mu^{P}(z)= \begin{cases}\mu\left(F_{1}\right)(z) & \text { for } z \in \Sigma_{0}, \\ 0 & \text { for } z \in \overline{\mathbb{C}} \backslash \Sigma_{0} .\end{cases}
$$

Let $F_{1}^{P}: \Sigma_{0}^{P} \rightarrow \overline{\mathbb{C}}$ be a quasiconformal map with dilatation $\mu^{P}$. Since $F_{1}^{P}$ is quasiconformal it has a unique continuous (in fact quasiconformal) extension to $\overline{\mathbb{C}}$; we will use the same notation for the extension.

We now define the fibration maps

$$
\begin{aligned}
\mathcal{P}: T\left(\Sigma_{0}\right) & \longrightarrow \widetilde{T}\left(\Sigma_{0}^{P}\right) \\
{\left[\Sigma_{0}, F_{1}, \Sigma_{1}\right] } & \longmapsto\left[\Sigma_{0}^{P}, F_{1}^{P}, \Sigma_{1}^{P},\left(F_{1}^{P} \circ \tau_{1}, \ldots, F_{1}^{P} \circ \tau_{n}\right)\right]
\end{aligned}
$$


and

$$
\begin{aligned}
\mathcal{F}: \widetilde{T}\left(\Sigma_{0}^{P}\right) & \longrightarrow T\left(\Sigma^{P}\right) \\
{\left[\Sigma_{0}^{P}, F_{1}^{P}, \Sigma_{1}^{P}, \boldsymbol{f}\right] } & \longmapsto\left[\Sigma_{0}^{P}, F_{1}^{P}, \Sigma_{1}^{P}\right] .
\end{aligned}
$$

We also require some results on the modular group; proofs and details can be found in 15 . The modular group $\operatorname{PModI}\left(\Sigma_{0}\right)$ consists of the set of quasiconformal self-maps of $\Sigma_{0}$ which are the identity on $\partial \Sigma_{0}$, modulo homotopy rel boundary. The "P" in "PMod" stands for "pure", which signifies that the self-maps fix the ordering of the boundary components. Given a quasiconformal $\rho: \Sigma_{0} \rightarrow \Sigma_{0}$ fixing the boundary, denote its equivalence class by $[\rho]$. The modular group $\operatorname{PModI}\left(\Sigma_{0}\right)$ acts on $T\left(\Sigma_{0}\right)$ via

$$
[\rho]^{*}\left[\Sigma_{0}, F_{1}, \Sigma_{1}\right]=\left[\Sigma_{0}, F_{1} \circ \rho^{-1}, \Sigma_{1}\right] .
$$

Let DB be the subgroup of PModI generated by Dehn twists around the boundary curves of $\Sigma_{0}$. It was proven in [15, Theorem 5.6] that

Theorem 3.3. $\mathcal{P}(u)=\mathcal{P}(v)$ if and only if there is $a[\rho] \in \mathrm{DB}$ such that $[\rho]^{*} u=v$.

Furthermore by [15, Corollary 6.2, Corollary 5.1]

Theorem 3.4. $\mathcal{F}$ and $\mathcal{P}$ are holomorphic.

Finally, we need one further result. Its statement is technical, but it is quite powerful for proving holomorphicity in situations which involve conformal welding, either implicitly or explicitly. Here, welding is implicit in the extension of the Beltrami differentials by 0 to the caps. The general result can be found in [17]; we specialize to the situation that $\Sigma_{0}^{P}$ is a punctured sphere. Some conditions relating to the normalization are added, which do not follow directly from the statement of the theorem in [17. For this reason we include a brief proof.

Theorem 3.5. Fix $n>3$. Let $\Sigma_{0}^{P}=\overline{\mathbb{C}} \backslash\left\{p_{1}, \ldots, p_{n}\right\}$ for points $p_{1}, \ldots, p_{n} \in \overline{\mathbb{C}}$. Let $d$ be the dimension of $T\left(\Sigma_{0}^{P}\right)$. Fix any $u=\left[\Sigma_{0}^{P}, F_{*}^{P}, \Sigma_{*}^{P}\right] \in T\left(\Sigma_{0}^{P}\right)$ and let $\left(\Sigma_{0}^{P}, F_{*}^{P}, \Sigma_{*}^{P}\right)$ be the unique representative such that $\Sigma_{*}^{P}$ is a sphere with punctures $\left(q_{1}, q_{2}, q_{3}, \ldots, q_{n}\right)$, where $q_{i}=p_{i}$ for $i=1,2,3$. Let $\boldsymbol{f}=\left(f_{1}, \ldots, f_{n}\right) \in \mathcal{O}^{\mathrm{qc}}\left(\Sigma_{*}^{P}\right)$ be a rigging on $\Sigma_{*}^{P}$, let $K_{i}$ be compact, non-overlapping sets on $\Sigma_{*}^{P}$ containing $p_{i}$ in their interiors, and let $V$ be as in Remark 2.9.

There is an open set $N \subseteq \mathbb{C}^{d}$ containing 0 and a map $\nu: N \times \overline{\mathbb{C}} \rightarrow \overline{\mathbb{C}}$ such that

(1) $\nu_{\epsilon}$ fixes $p_{1}, p_{2}$, and $p_{3}$ (where $\nu_{\epsilon}(z)=\nu(\epsilon, z)$ ),

(2) for fixed $\epsilon, \nu(\epsilon, z)$ is quasiconformal on $\overline{\mathbb{C}}$ and holomorphic on $\bigcup_{i=1}^{n} K_{i}$ (that is, one-to-one and meromorphic),

(3) $\nu(\epsilon, z)$ is holomorphic in $\epsilon$ for any fixed $z$, and

(4) denoting $\nu_{\epsilon}(z)=\nu(\epsilon, z)$, the map $\epsilon \mapsto\left[\Sigma_{0}, \nu_{\epsilon} \circ F_{*}^{P}, \nu_{\epsilon}\left(\Sigma_{*}^{P}\right)\right]$ is a local biholomorphic coordinate system on $T\left(\Sigma_{0}^{P}\right)$ onto a neighborhood of $u$.

Furthermore, for this map $\nu_{\epsilon}$,

(5) the map

$$
\begin{aligned}
\Psi: N \times V & \longrightarrow \widetilde{T}\left(\Sigma_{0}^{P}\right) \\
(\epsilon, \boldsymbol{f}) & \longmapsto\left[\Sigma_{0}^{P}, \nu_{\epsilon} \circ F_{*}^{P}, \nu_{\epsilon}\left(\Sigma_{*}^{P}\right), \nu_{\epsilon} \circ \boldsymbol{f}\right]
\end{aligned}
$$

is a local biholomorphic coordinate system on $\widetilde{T}\left(\Sigma_{0}^{P}\right)$. 
Proof. By a result of F. Gardiner [6] (see also [10, Theorem 4.3.2]) there is a quasiconformal map $\nu_{\epsilon}$ on $\Sigma_{*}^{P}$ which is quasiconformal on $\overline{\mathbb{C}}$ and holomorphic on $\bigcup_{i=1}^{n} K_{i}$ such that $\epsilon \rightarrow\left[\Sigma_{*}^{P}, \nu_{\epsilon}, \nu_{\epsilon}\left(\Sigma_{*}^{P}\right)\right]$ form holomorphic coordinates for $T\left(\Sigma_{*}^{P}\right)$ in a neighborhood of $\left[\Sigma_{*}^{P}\right.$, Id, $\left.\Sigma_{*}^{P}\right]$. Note that in Gardiner's construction the Beltrami differential of $\nu_{\epsilon}$ is given explicitly and depends holomorphically on $\epsilon$; we will require this fact ahead. Since change of base point in Teichmüller space is biholomorphic, we obtain that $\epsilon \mapsto\left[\Sigma_{0}, \nu_{\epsilon} \circ F_{*}^{P}, \nu_{\epsilon}\left(\Sigma_{*}^{P}\right)\right]$ are coordinates on $T\left(\Sigma^{P}\right)$ for a neighborhood of $\left[\Sigma_{0}, F_{*}^{P}, \Sigma_{*}^{P}\right]$ ([17, Theorem 2.17 and text immediately following]). Thus we have that (2) and (4) hold. Claim (5) is stated explicitly and proved in [17, proof of Theorem 4.1]. Note that there the map $\Psi$ is labelled $H$.

Since $\nu_{\epsilon}\left(\Sigma_{*}^{P}\right)$ is quasiconformally equivalent to a punctured sphere, by the uniformization theorem it is biholomorphic to the punctured sphere. Thus we may normalize $\nu_{\epsilon}$ so that $\nu_{\epsilon}$ is a map of the punctured sphere which fixes $q_{i}=p_{i}$ for $i=1,2,3$. Thus we obtain property (1), and the normalization obviously does not affect property (2). Since the normalization preserves the equivalence class in both $T\left(\Sigma^{P}\right)$ and $\tilde{T}\left(\Sigma^{P}\right)$, the maps in properties (4) and (5) are unchanged and thus (4) and (5) continue to hold.

Finally, recall that the dilatation of $\nu_{\epsilon}$ depends holomorphically on $\epsilon$; property (3) thus is a classical property of solutions to the Beltrami equation with holomorphically varying dilatation [10, Theorem 1.2.11, p. 38].

3.2. Representation of Teichmüller space by Grunsky matrices. We return to the problem of defining the period mapping. Assume that $n>3$ and recall the definitions of $\widehat{\mathbf{G r}}$ and $\mathfrak{B}(n)$ from equations (2.1) and (2.5), respectively. We define

$$
\begin{aligned}
\tilde{\Pi}: \widetilde{T}\left(\Sigma_{0}^{P}\right) & \longrightarrow T\left(\Sigma_{0}^{P}\right) \times \mathfrak{B}(n) \\
\left(\Sigma_{0}^{P}, F_{1}^{P}, \Sigma_{1}^{P}, \boldsymbol{f}\right) & \longmapsto\left(\left[\Sigma_{0}^{P}, F_{1}^{P}, \Sigma_{1}^{P}\right], \widehat{\mathbf{G r}}(\boldsymbol{f})\right) .
\end{aligned}
$$

To see that this is well-defined, observe that if

$$
\left(\Sigma_{0}^{P}, F_{1}^{P}, \Sigma_{1}^{P}, \boldsymbol{f}_{1}\right) \sim\left(\Sigma_{0}^{P}, F_{2}^{P}, \Sigma_{2}^{P}, \boldsymbol{f}_{2}\right),
$$

then there is a Möbius transformation $\sigma: \overline{\mathbb{C}} \rightarrow \overline{\mathbb{C}}$ taking the punctures of $\Sigma_{1}^{P}$ to those of $\Sigma_{2}^{P}$ and such that $\boldsymbol{f}_{2}=\sigma \circ \boldsymbol{f}_{1}$. Thus $\left[\Sigma_{0}^{P}, F_{1}^{P}, \Sigma_{1}^{P}\right]=\left[\Sigma_{0}^{P}, F_{2}^{P}, \Sigma_{2}^{P}\right]$ by the definition of Teichmüller equivalence, and $\widehat{\mathbf{G r}}\left(\boldsymbol{f}_{1}\right)=\widehat{\mathbf{G r}}\left(\boldsymbol{f}_{2}\right)$ by Theorem 2.5. Thus $\tilde{\Pi}$ is well-defined.

Define also $\Pi=\tilde{\Pi} \circ \mathcal{P}$. In that case $\Pi$ is given by

$$
\begin{aligned}
\Pi: T\left(\Sigma_{0}\right) & \longrightarrow T\left(\Sigma_{0}^{P}\right) \times \mathfrak{B}(n) \\
{\left[\Sigma_{0}, F_{1}, \Sigma_{1}\right] } & \longmapsto\left(\left[\Sigma_{0}^{P}, F_{1}^{P}, \Sigma_{1}^{P}\right], \widehat{\mathbf{G r}}(\boldsymbol{f})\right),
\end{aligned}
$$

where

$$
\boldsymbol{f}=\left(F_{1}^{P} \circ \tau_{1}, \ldots, F_{1}^{P} \circ \tau_{n}\right)
$$

and $F_{1}^{P}$ is determined from $F_{1}$ via extending the Beltrami differential of $F_{1}$ by zero on the caps, as specified in the previous section. Since $\mathcal{P}$ is well-defined 15 and $\tilde{\Pi}$ is well-defined, so is $\Pi$. Denote the two components of $\Pi$ by $\Pi_{1}: T\left(\Sigma_{0}\right) \rightarrow T\left(\Sigma_{0}^{P}\right)$ and $\Pi_{2}: T\left(\Sigma_{0}\right) \rightarrow \mathfrak{B}(n)$, and similarly for $\tilde{\Pi}$. 
If $n=1, n=2$, or $n=3$, the Teichmüller space of $\Sigma_{0}^{P}$ reduces to a point. In those cases, we define $\Pi$ and $\tilde{\Pi}$ as maps into $\mathfrak{B}(n)$ :

$$
\begin{aligned}
\tilde{\Pi}: \widetilde{T}\left(\Sigma_{0}^{P}\right) & \longrightarrow \mathfrak{B}(n) \\
\left(\Sigma_{0}^{P}, F_{1}^{P}, \Sigma_{1}^{P}, \boldsymbol{f}\right) & \longmapsto \widehat{\mathbf{G r}}(\boldsymbol{f})
\end{aligned}
$$

and

$$
\begin{gathered}
\Pi: T\left(\Sigma_{0}\right) \longrightarrow \mathfrak{B}(n) \\
{\left[\Sigma_{0}, F_{1}, \Sigma_{1}\right] \longmapsto \widehat{\mathbf{G r}}(\boldsymbol{f}) .}
\end{gathered}
$$

The case that $n=1$ was considered and shown to be holomorphic by Takhtajan and Teo 23 .

Remark 3.6. In the cases that $n$ is equal to 1,2 , or 3 , the equivalence relation on $\widetilde{T}\left(\Sigma_{0}^{P}\right)$ says that two elements $\left(\Sigma_{0}^{P}, F_{1}^{P}, \Sigma_{1}^{P}, \boldsymbol{f}\right)$ and $\left(\Sigma_{0}^{P}, F_{2}^{P}, \Sigma_{2}^{P}, \boldsymbol{g}\right)$ are equivalent if and only if there is some conformal map $\sigma: \Sigma_{1}^{P} \rightarrow \Sigma_{2}^{P}$ such that $\sigma \circ f_{i}=g_{i}$ for $i=1, \ldots, n$.

Remark 3.7. It is clear that $\Pi$ and $\tilde{\Pi}$ depend on $\tau$.

In order to prove the main theorem, we require a technical lemma. Recall that the complex structure on $\mathcal{O}^{\text {qc }}$ is induced by $\mathbb{C} \oplus A_{1}^{\infty}\left(\mathbb{D}^{+}\right)$.

Lemma 3.8. Let $E$ be an open subset of $\mathbb{C}$ containing 0 , and let $\Delta$ be an open subset of $\mathbb{C}$. Let $M: \Delta \times E \rightarrow \mathbb{C}$ be a map which is holomorphic in both variables and injective in the second variable, and let $M_{\epsilon}(z)=M(\epsilon, z)$. Let $\psi \in \mathcal{O}^{\mathrm{qc}}$ satisfy $\psi\left(\mathbb{D}^{+}\right)^{\mathrm{cl}} \subseteq E$. Then the map $Q: \Delta \mapsto \mathcal{O}^{\mathrm{qc}}$ defined by $Q(\epsilon)=M_{\epsilon} \circ \psi$ is holomorphic $($ in $\epsilon)$.

Proof. Define $\mathcal{A}(f)=f^{\prime \prime} / f^{\prime}$. We need to show that for fixed $\psi, \mathcal{A}\left(M_{\epsilon} \circ \psi\right)$ and $\left(M_{\epsilon} \circ \psi\right)^{\prime}(0)$ are holomorphic in $\epsilon$. The second claim follows from the fact that the $z$-derivatives of all orders of $M_{\epsilon}$ are holomorphic in $\epsilon$ for fixed $z$.

To prove holomorphicity of $\epsilon \mapsto \mathcal{A}\left(M_{\epsilon} \circ \psi\right)$, it is enough to show weak holomorphicity and local boundedness in the $A_{1}^{\infty}\left(\mathbb{D}^{+}\right)$norm [7; that is, to show local boundedness and that for some set of separating continuous functionals $\{\alpha\}$ in the dual of $A_{1}^{\infty}\left(\mathbb{D}^{+}\right), \alpha \circ \mathcal{A}\left(M_{\epsilon} \circ \psi\right)$ is holomorphic for all $\alpha$. Let $e_{z}$ be the point evaluation function $e_{z} \psi=\psi(z)$. These are continuous on $A_{1}^{\infty}\left(\mathbb{D}^{+}\right)$and obviously separating on any open set. Since

$$
\mathcal{A}\left(M_{\epsilon} \circ \psi\right)=\mathcal{A}\left(M_{\epsilon}\right) \circ \psi \cdot \psi^{\prime}+\mathcal{A}(\psi),
$$

clearly $e_{z}\left(\mathcal{A}\left(M_{\epsilon} \circ f\right)\right)$ is holomorphic in $\epsilon$.

Next, let $F \subseteq E$ be a simply connected open set such that $\psi\left(\mathbb{D}^{+}\right)^{\mathrm{cl}} \subset F$. Let $\lambda_{F}(z)^{2}$ denote the hyperbolic line element on $F$ (that is, $\lambda_{F}(z)=\left|g^{\prime}(z)\right| /\left(1-|g(z)|^{2}\right)$ for any biholomorphism $\left.g: F \rightarrow \mathbb{D}^{+}\right)$. Since $\psi\left(\mathbb{D}^{+}\right)^{\text {cl }}$ is compactly contained in $F, 1 / \lambda_{F}(\psi(z))$ is bounded below on $\mathbb{D}^{+}$. Thus by the Schwarz lemma, there is a constant $C$ such that

$$
\left(1-|z|^{2}\right)\left|\psi^{\prime}(z)\right| \leq \frac{1}{\lambda_{F}(\psi(z))} \leq C
$$

for all $z \in \mathbb{D}^{+}$. 
It remains to show that $\mathcal{A}\left(M_{\epsilon} \circ \psi\right)$ is locally bounded. Equality (3.5) yields that for any fixed $\epsilon$

$$
\begin{aligned}
\left\|\mathcal{A}\left(M_{\epsilon} \circ \psi\right)\right\|_{A_{1}^{\infty}\left(\mathbb{D}^{+}\right)} \leq \sup _{z \in \mathbb{D}^{+}}\left|\mathcal{A}\left(M_{\epsilon}\right) \circ \psi(z)\right| \sup _{z \in \mathbb{D}^{+}}\left(1-|z|^{2}\right)\left|\psi^{\prime}(z)\right| \\
+\sup _{z \in \mathbb{D}^{+}}\left(1-|z|^{2}\right)|\mathcal{A}(\psi)(z)| .
\end{aligned}
$$

Since $\mathcal{A}\left(M_{\epsilon}\right)$ is jointly holomorphic in $\epsilon$ and $z$ and $\psi(\mathbb{D})^{\mathrm{cl}} \subseteq E$ for any fixed $\epsilon_{0}$, there is a compact set $D$ containing $\epsilon_{0}$ such that $\left|\mathcal{A}\left(M_{\epsilon}\right)\right|$ is bounded on $\psi\left(\mathbb{D}^{+}\right)$by a constant independent of $\epsilon \in D$. Using (3.6) and the fact that $\mathcal{A}(\psi)$ is in $A_{1}^{\infty}\left(\mathbb{D}^{+}\right)$ we obtain that $\mathcal{A}\left(M_{\epsilon} \circ \psi\right)$ is locally bounded, which completes the proof.

We now prove the main theorem.

Theorem 3.9. $\Pi$ and $\tilde{\Pi}$ are holomorphic.

Proof. Since $\mathcal{P}$ and $\tilde{\Pi}_{1}=\mathcal{F}$ are holomorphic by Theorem 3.4 , it suffices to show that $\tilde{\Pi}_{2}$ is holomorphic.

Fix an arbitrary point $\left[\Sigma_{0}, F_{*}^{P}, \Sigma_{*}^{P}, \boldsymbol{f}^{0}\right] \in \widetilde{T}\left(\Sigma_{0}\right)$. We will show that $\tilde{\Pi}$ is holomorphic at this point. Choose the representative $\Sigma_{*}^{P}=\overline{\mathbb{C}} \backslash\left\{p_{1}, \ldots, p_{n}\right\}$, and $\boldsymbol{f}^{0}=\left(f_{1}^{0}, \ldots, f_{n}^{0}\right) \in \mathcal{O}^{\mathrm{qc}}\left(\Sigma_{*}^{P}\right)$. Let $K_{i}, W, V$, and $G$ be as in Remark [2.9] and choose $N$ as in Theorem 2.11. If $n>3$, by Theorem 3.5 and Remark 2.9 it is enough to show that $\widetilde{\Pi}_{2} \circ \Psi \circ(\operatorname{Id} \times G)$ is holomorphic, where Id is the identity on $N$. In the cases that $n=2$ or $n=3$, it automatically reduces to this. The explicit formula is

$$
\widetilde{\Pi}_{2} \circ \Psi \circ(\operatorname{Id} \times G)\left(\epsilon, g_{1}, \ldots, g_{n}\right)=\widehat{\mathbf{G r}}\left(\nu_{\epsilon}\left(g_{1}+p_{1}\right), \ldots, \nu_{\epsilon}\left(g_{n}+p_{n}\right)\right) .
$$

By Hartog's theorem [9] it is enough to show separate holomorphicity in $\epsilon$ and in $\mathcal{O}^{\text {qc }}(n)$.

First we fix $\epsilon=0$. In this case, we have that

$$
\widetilde{\Pi}_{2} \circ \Psi \circ(\operatorname{Id} \times G)\left(0, g_{1}, \ldots, g_{n}\right)=\widehat{\mathbf{G r}}\left(g_{1}+p_{1}, \ldots, g_{n}+p_{n}\right) .
$$

This is holomorphic in $\mathcal{O}^{\text {qc }}(n)$ by applying Theorem 2.11 with fixed $\left(a_{1}, \ldots, a_{n}\right)$.

Now fix $\left(g_{1}^{0}, \ldots, g_{n}^{0}\right)=G^{-1}\left(\boldsymbol{f}^{0}\right)=\left(f_{1}^{0}-p_{1}, \ldots, f_{n}^{0}-p_{n}\right)$ and vary $\epsilon$. In this case we have

$$
\widetilde{\Pi}_{2} \circ \Psi \circ(\operatorname{Id} \times G)\left(\epsilon, g_{1}^{0}, \ldots, g_{n}^{0}\right)=\widehat{\mathbf{G r}}\left(\nu_{\epsilon}\left(f_{1}^{0}\right), \ldots, \nu_{\epsilon}\left(f_{n}^{0}\right)\right) .
$$

If we set $\hat{\nu}_{i}(\epsilon, z)=\nu_{\epsilon}(z)-\nu_{\epsilon}\left(p_{i}\right)$, then we can write

$$
\widehat{\mathbf{G r}}\left(\nu_{\epsilon}\left(f_{1}^{0}\right), \ldots, \nu_{\epsilon}\left(f_{n}^{0}\right)\right)=H\left(\hat{\nu}_{1}\left(\epsilon, f_{1}^{0}\right), \ldots, \hat{\nu}_{n}\left(\epsilon, f_{n}^{0}\right), \nu_{\epsilon}\left(p_{1}\right), \ldots, \nu_{\epsilon}\left(p_{n}\right)\right),
$$

where $H$ is defined in Theorem 2.11. Now by Theorem 3.5 $\epsilon \mapsto \nu_{\epsilon}\left(p_{i}\right)$ is holomorphic in $\epsilon$, and by Lemma 3.8 combined with Hartogs' theorem on separate holomorphicity in finitely many variables, the map from $N$ to $\mathcal{O}^{\text {qc }}$ given by

$$
\epsilon \longmapsto \hat{\nu}_{i}\left(\epsilon, f_{i}^{0}\right)
$$

is holomorphic. This together with Theorem 2.11 shows that $\widetilde{\Pi}_{2} \circ \Psi \circ(\operatorname{Id} \times G)$ is holomorphic at $\left(0, f_{1}^{0}, \ldots, f_{n}^{0}\right)$. Since the point was arbitrary this completes the proof.

The next theorem shows that the map $\tilde{\Pi}$ is injective, and $\Pi$ is nearly so.

Theorem 3.10. $\tilde{\Pi}(u)=\tilde{\Pi}(v)$ if and only if $u=v$. $\Pi(u)=\Pi(v)$ if and only if there is $a[\rho] \in \mathrm{DB}$ such that $\rho^{*} u=v$. 
Proof. The second claim follows from the first by Theorem 3.3 and the fact that $\tilde{\Pi} \circ \mathcal{P}=\Pi$.

Now assume that $\tilde{\Pi}(p)=\left(\left[\Sigma_{0}^{P}, F_{1}^{P}, \Sigma_{1}^{P}\right], S\right)$. For $S=\widehat{\mathbf{G r}}(\boldsymbol{f}) \in \mathfrak{B}(n)$, the kernel functions of the block operators $\widehat{G r}_{i j}$ are uniquely determined by $S$ for all $i, j$. To see this, one need only apply the operator to the Bergman kernel for $A^{2}\left(\mathbb{D}^{+}\right)$in place of $\hat{\mathbf{R}} h$ in Theorem 2.3 .

The proof proceeds in cases, depending on whether $n=1, n=2$, or $n \geq 3$. If $n=1$, as already observed, the function

$$
\frac{1}{(\zeta-z)^{2}}-\frac{f_{1}^{\prime}(\zeta) f_{1}^{\prime}(z)}{\left(f_{1}(\zeta)-f_{1}(z)\right)^{2}}
$$

is uniquely determined by $S$. Now letting $\zeta \rightarrow z$, identity (3.7) in Bergman-Schiffer's paper 2 yields that this quantity tends to one-sixth of the Schwarzian derivative of $f_{1}$. Thus the Schwarzian of $f_{1}$ is uniquely determined by $S$, and therefore $f_{1}$ is uniquely determined up to post-composition by a Möbius transformation. The claim now follows from Remark 3.6.

Now assume that $n=2$, and that $\tilde{\Pi}(u)=\left(\left[\Sigma_{0}^{P}, F_{1}^{P}, \Sigma_{1}^{P}\right], S\right)$ is given. Let $\left(\Sigma_{0}^{P}, F_{1}^{P}, \Sigma_{1}^{P}, \boldsymbol{f}\right)$ be any representative of $u$, where $\boldsymbol{f}=\left(f_{1}, f_{2}\right)$. By post-composing $\Sigma_{1}^{P}, F_{1}^{P}, f_{1}$, and $f_{2}$ simultaneously by a Möbius transformation $\sigma$, we can assume that $f_{1}$ and $f_{2}$ are normalized so that $f_{1}(0)=0, f_{1}^{\prime}(0)=1$, and $f_{2}(0)=1$ (any fixed value will do). If it can be shown that this uniquely determines $f_{1}$ and $f_{2}$, then by Remark 3.6 it will follow that $\widetilde{\Pi}(u)=\widetilde{\Pi}(v) \Rightarrow u=v$.

By the first paragraph of the proof, we have that the kernel function of $\operatorname{Gr}_{12}(f)$,

$$
\frac{f_{1}^{\prime}(\zeta) f_{2}^{\prime}(z)}{\left(f_{1}(\zeta)-f_{2}(z)\right)^{2}},
$$

is uniquely determined by $\widetilde{\Pi}(u)$. Setting $\zeta=z=0$ yields that $f_{2}^{\prime}(0)$ is uniquely determined. Differentiating (3.8) with respect to $z$, we see that

$$
\frac{f_{1}^{\prime}(\zeta) f_{2}^{\prime \prime}(z)\left(f_{1}(\zeta)-f_{2}(z)\right)+2 f_{1}^{\prime}(\zeta) f_{2}^{\prime}(z)^{2}}{\left(f_{1}(\zeta)-f_{2}(z)\right)^{3}}
$$

is uniquely determined, and setting $\zeta=z=0$, one can also determine $f_{2}^{\prime \prime}(0)$ uniquely. The same argument applied to $\operatorname{Gr}_{21}(f)$ shows that $f_{1}^{\prime \prime}(0)$ is determined uniquely, and applying the considerations in the first paragraph to $\operatorname{Gr}_{11}(f)$ and $\mathrm{Gr}_{22}(f)$ shows that the Schwarzians of $f_{1}$ and $f_{2}$ are determined by $\widetilde{\Pi}(u)$. Since we have determined $f_{i}(0), f_{i}^{\prime}(0), f_{i}^{\prime \prime}(0)$ for $i=1,2$, the $f_{i}$ 's are uniquely determined and the claim follows.

Now we consider the case that $n \geq 3$; again assume $\tilde{\Pi}(u)=\left(\left[\Sigma_{0}^{P}, F_{1}^{P}, \Sigma_{1}^{P}\right], S\right)$ is given and $\left(\Sigma_{0}^{P}, F_{1}^{P}, \Sigma_{1}^{P}, \boldsymbol{f}\right)$ is any representative of $p$. By post-composing $\Sigma_{1}^{P}$, $F_{1}^{P}$, and $\boldsymbol{f}$ simultaneously by a Möbius transformation $\sigma$, we can assume that $f_{1}$, $f_{2}$, and $f_{3}$ are normalized so that $f_{1}(0)=0, f_{2}(0)=1$, and $f_{3}(0)=-1$ (again, any fixed values will do). If $k \geq 3$, then the remaining values of the points $f_{k}(0)$ for $k \neq 1,2,3$ are uniquely determined by the values of $F_{1}^{P}\left(p_{k}\right)$. This is because these values are determined by the Teichmüller equivalence class $\left[\Sigma_{0}^{P}, F_{1}^{P}, \Sigma_{1}^{P}\right]$ up to post-composition by $\sigma$, and $\sigma$ is uniquely determined by the normalizations above. As in the $n=2$ case, by Remark 3.6 it is enough to show that $f_{1}, \ldots, f_{n}$ are now uniquely determined. 
Arguing as in the $n=2$ case, for any $i \neq j, \widetilde{\Pi}(u)$ uniquely determines

$$
\frac{f_{i}^{\prime}(\zeta) f_{j}^{\prime}(z)}{\left(f_{i}(\zeta)-f_{j}(z)\right)^{2}} .
$$

Again setting $\zeta=z=0$, we see that for $i \neq j$, all pairwise products $f_{i}^{\prime}(0) f_{j}^{\prime}(0)$ are uniquely determined. By an easy algebraic argument, fixing any three pairwise distinct values $i, j$, and $k$, the resulting three products $f_{i}^{\prime}(0) f_{j}^{\prime}(0), f_{j}^{\prime}(0) f_{k}^{\prime}(0)$, and $f_{k}^{\prime}(0) f_{i}^{\prime}(0)$ uniquely determine $f_{i}^{\prime}(0), f_{j}^{\prime}(0)$, and $f_{k}^{\prime}(0)$. Since $i, j, k$ are arbitrary we have shown that $f_{i}^{\prime}(0)$ is determined uniquely for $i=1, \ldots, n$.

By differentiating the kernels $\widehat{\operatorname{Gr}}_{i j}$ twice with respect to $z$ and setting $\zeta=z=0$ as in the $n=2$ case, we uniquely determine $f_{i}^{\prime \prime}(0)$ for all $i=1, \ldots, n$. Also once again, we have that $\mathrm{Gr}_{i i}$ uniquely determines the Schwarzian of $f_{i}$ for all $i$. Thus the $f_{i}$ 's are uniquely determined, and this completes the proof.

Remark 3.11. One can of course compose the map $\Pi$ by the classical period map on $T\left(\Sigma^{P}\right)$ to obtain a full embedding of $T(\Sigma)$ by a period mapping.

\section{REFERENCES}

[1] Lars V. Ahlfors and Leo Sario, Riemann surfaces, Princeton Mathematical Series, No. 26, Princeton University Press, Princeton, N.J., 1960. MR.0114911

[2] S. Bergman and M. Schiffer, Kernel functions and conformal mapping, Compositio Math. 8 (1951), 205-249. MR0039812

[3] Abdullah Çavuş, Approximation by generalized Faber series in Bergman spaces on finite regions with a quasiconformal boundary, J. Approx. Theory 87 (1996), no. 1, 25-35, DOI 10.1006/jath.1996.0090. MR1410610

[4] Soo Bong Chae, Holomorphy and calculus in normed spaces, Monographs and Textbooks in Pure and Applied Mathematics, vol. 92, Marcel Dekker, Inc., New York, 1985. With an appendix by Angus E. Taylor. MR788158

[5] Peter L. Duren, Univalent functions, Grundlehren der Mathematischen Wissenschaften [Fundamental Principles of Mathematical Sciences], vol. 259, Springer-Verlag, New York, 1983. MR708494

[6] Frederick P. Gardiner, Schiffer's interior variation and quasiconformal mapping, Duke Math. J. 42 (1975), 371-380. MR.0382637

[7] K.-G. Grosse-Erdmann, A weak criterion for vector-valued holomorphy, Math. Proc. Cambridge Philos. Soc. 136 (2004), no. 2, 399-411, DOI 10.1017/S0305004103007254. MR2040581

[8] J. A. Hummel, Inequalities of Grunsky type for Aharonov pairs, J. Analyse Math. 25 (1972), 217-257, DOI 10.1007/BF02790039. MR0311893

[9] Jorge Mujica, Complex analysis in Banach spaces: Holomorphic functions and domains of holomorphy in finite and infinite dimensions; Notas de Matemática [Mathematical Notes], 107, North-Holland Mathematics Studies, vol. 120, North-Holland Publishing Co., Amsterdam, 1986. MR 842435

[10] Subhashis Nag, The complex analytic theory of Teichmüller spaces, Canadian Mathematical Society Series of Monographs and Advanced Texts, John Wiley \& Sons, Inc., New York, 1988. A Wiley-Interscience Publication. MR927291

[11] Subhashis Nag, A period mapping in universal Teichmüller space, Bull. Amer. Math. Soc. (N.S.) 26 (1992), no. 2, 280-287, DOI 10.1090/S0273-0979-1992-00273-2. MR.1121571

[12] Subhashis Nag and Dennis Sullivan, Teichmüller theory and the universal period mapping via quantum calculus and the $H^{1 / 2}$ space on the circle, Osaka J. Math. 32 (1995), no. 1, 1-34. MR 1323099

[13] Christian Pommerenke, Univalent functions, Studia Mathematica/Mathematische Lehrbücher, Band XXV, Vandenhoeck \& Ruprecht, Göttingen, 1975. With a chapter on quadratic differentials by Gerd Jensen. MR.0507768

[14] David Aubrey Alfred Radnell, Schiffer variation in Teichmuller space, determinant line bundles and modular functors, ProQuest LLC, Ann Arbor, MI, 2003. Thesis (Ph.D.)-Rutgers The State University of New Jersey - New Brunswick. MR2705159 
[15] David Radnell and Eric Schippers, Quasisymmetric sewing in rigged Teichmüller space, Commun. Contemp. Math. 8 (2006), no. 4, 481-534, DOI 10.1142/S0219199706002210. MR2258875

[16] David Radnell and Eric Schippers, A complex structure on the set of quasiconformally extendible non-overlapping mappings into a Riemann surface, J. Anal. Math. 108 (2009), 277291, DOI 10.1007/s11854-009-0025-0. MR2544761

[17] David Radnell and Eric Schippers, Fiber structure and local coordinates for the Teichmüller space of a bordered Riemann surface, Conform. Geom. Dyn. 14 (2010), 14-34, DOI 10.1090/S1088-4173-10-00206-7. MR2593332

[18] David Radnell, Eric Schippers, and Wolfgang Staubach, Dirichlet spaces of domains bounded by quasicircles, to appear in Commun. Contemp. Math., preprint arXiv:1705.01279v2.

[19] Eric Schippers and Wolfgang Staubach, Harmonic reflection in quasicircles and wellposedness of a Riemann-Hilbert problem on quasidisks, J. Math. Anal. Appl. 448 (2017), no. 2, 864-884, DOI 10.1016/j.jmaa.2016.11.041. MR3582264

[20] Eric Schippers and Wolfgang Staubach, Riemann boundary value problem on quasidisks, Faber isomorphism and Grunsky operator, Complex Anal. Oper. Theory 12 (2018), no. 2, 325-354, DOI 10.1007/s11785-016-0598-4. MR3756161

[21] YuLiang Shen, Faber polynomials with applications to univalent functions with quasiconformal extensions, Sci. China Ser. A 52 (2009), no. 10, 2121-2131, DOI 10.1007/s11425-0090062-2. MR 2550270

[22] P. K. Suetin, Series of Faber polynomials, Analytical Methods and Special Functions, vol. 1, Gordon and Breach Science Publishers, Amsterdam, 1998. Translated from the 1984 Russian original by E. V. Pankratiev [E. V. Pankrat'ev]. MR1676281

[23] Leon A. Takhtajan and Lee-Peng Teo, Weil-Petersson metric on the universal Teichmüller space, Mem. Amer. Math. Soc. 183 (2006), no. 861, viii+119, DOI 10.1090/memo/0861. $\operatorname{MR} 2251887$

Department of Mathematics and Systems Analysis, Aalto University, P.O. Box 11100, FI-00076 AALTO, Finland

Email address: david.radnell@aalto.fi

Department of Mathematics, University of Manitoba, Winnipeg, Manitoba, R3T 2N2, CANADA

Email address: eric.schippers@umanitoba.ca

Department of Mathematics, Uppsala University, Box 480, 75106 Uppsala, Sweden

Email address: wulf@math.uu.se 